\title{
Influence of Reaction Heat on Time Dependent Processes in a Chemically Reacting binary mixture
}

\author{
Wilson Marques Jr.*, Gilberto M. Kremer* and \\ Ana Jacinta Soares ${ }^{\dagger}$ \\ * Departamento de Física, Universidade Federal do Paraná, Curitiba, Brazil \\ ${ }^{\dagger}$ Centro de Matemática, Universidade do Minho, Braga, Portugal
}

\begin{abstract}
In this paper we study time dependent problems, like the propagation of sound waves or the behavior of small local wave disturbances induced by spontaneous internal fluctuations, in a binary mixture undergoing a chemical reaction of type $A+A \rightleftharpoons B+B$. The study is developed at the hydrodynamic Euler level, in a chemical regime of fast reactive process in which the chemical reaction is close to its final equilibrium state. The hydrodynamic state of the mixture is described by the balance equations for the mass densities of both constituents $A$ and $B$, together with the conservation laws for the momentum and total energy of the mixture. The progress of the chemical reaction is specified by an Arrhenius-type reaction rate which defines the net balance between production and consumption of each constituent. Assuming that the considered time dependent problems induce weak macroscopic deviations, the hydrodynamic equations are linearized through a normal mode expansion of the state variables around the equilibrium state. From the dispersion relation of the normal modes, we determine the free and forced phase velocities as well as the attenuation coefficients of the waves. We show that the dispersion and absorption of these waves depend explicitly on the heat of the chemical reaction, the concentrations of the constituents and the activation energy through the exponential factor of Arrhenius law.
\end{abstract}

Keywords: Boltzmann equation, Chemical reactions, Sound propagation

PACS: $47.45 . \mathrm{Ab}, 51.10 .+\mathrm{y}, 47.70 . \mathrm{Fw}, 43.20 . \mathrm{Bi}$

\section{INTRODUCTION}

Chemically reacting mixtures are widely investigated in literature due to their central role in many industrial applications, such as combustion processes, plasma physics and chemical reactors. In particular, many contributions have been proposed oriented to theoretical and formal studies as well as to the modeling of reactive systems, transport properties and non-equilibrium effects induced by the chemical process [1]-[4]. The propagation of sound waves and the behavior of small local wave disturbances in chemically reacting mixtures constitute interesting research topics which allow to investigate the interaction of the chemical reaction with the time evolution of the mixture. Different sound regimes have been investigated for reacting mixtures, based on both hydrodynamic equations and kinetic theory approaches, and several numerical simulations have been performed to describe the main features of the above mentioned physical problems. Here we quote, among others, the papers [5]-[10]. where some studies have been carried out to analyze sound propagation problems, light scattering phenomena, the dynamics of small perturbations and shock waves.

In the present work we consider a mixture of ideal gases of two constituents, $A$ and $B$, undergoing a simple reversible reaction of type $A+A \rightleftharpoons B+B$. Starting from a kinetic approach of the reactive mixture, we consider the hydrodynamic equations at the Euler level - i.e., a mixture where effects due to viscosity, thermal conductivity and diffusion are neglected - in a chemical regime of fast reactive process in which the chemical reaction is close to its final equilibrium state. The rate of the chemical reaction follows an Arrhenius law and the chemical potentials, with internal variables associated with the formation energy, do not reflect internal degrees of freedom, like rotational and vibrational effects.

Since we are interested in the effect of the reaction heat on the dispersion and attenuation of wave propagation, we develop a theoretical treatment of time dependent processes in an Eulerian reactive mixture. More precisely, we study the propagation of sound waves and the dynamics of small perturbations induced by spontaneous internal fluctuations. The mathematical analysis of these problems is based on the assumption that the perturbations have small amplitudes

${ }^{1}$ Dedicated to Professor Bernie D. Shizgal, on the occasion of his 70th birthday. 
so that a linearized theory is used to describe the dynamics of the disturbances induced in the state variables of the mixture. Then a normal mode expansion around an equilibrium state characterized by constant values for the constituent's mass densities and total energy of the mixture, and vanishing mixture velocity is considered and the dispersion relation of the normal modes is explicitly derived. The phase velocities and attenuation coefficients of forced waves are evaluated in the low-frequency limit, exhibiting an explicit dependence on the heat of the chemical reaction and constituent's concentrations. Finally two hydrodynamic modes and one kinetic mode of free wave propagation are obtained in the small wavenumber limit and, again, the spectrum of the wave disturbances is characterized in terms of the reaction heat and constituent's concentrations.

\section{HYDRODYNAMIC EQUATIONS FOR A REACTIVE MIXTURE}

We consider a chemically reacting binary mixture of ideal gases whose constituents - denoted by $A_{1}$ and $A_{2}-$ undergo a simple symmetric reversible reaction $A_{1}+A_{1} \rightleftharpoons A_{2}+A_{2}$. The molecules of the constituents are characterized by the formation (or binding) energies $\mathrm{e}_{1}$ and $\mathrm{e}_{2}$, by the same masses $m_{1}=m_{2}=m$ and same diameters $\mathrm{d}_{1}=\mathrm{d}_{2}=\mathrm{d}$. Chemical reactions of this kind may be considered as the first stage of the Lindemann-Hinshelwood mechanism for reactions of isomers [11].

The basic fields of the considered theory are the partial mass densities of the constituents $\rho_{1}$ and $\rho_{2}$, the momentum density $\rho \mathbf{v}$ and the total energy $\rho e$ of the mixture, where $\rho=\rho_{1}+\rho_{2}$ denotes the mixture mass density. The hydrodynamic equations describing the space-time evolution of the mixture are the balance equations for the mass densities $\rho_{1}$ and $\rho_{2}$ and the conservation laws for the momentum density $\rho v$ and total energy $\rho e$ of the mixture. In the case of an Eulerian mixture - where the diffusion fluxes, heat flux, shear and non-equilibrium stresses are absent - the one-space dimensional balance equations can be written in matrix form as

$$
\frac{\partial \mathbf{U}}{\partial t}+\frac{\partial \mathbf{F}(\mathbf{U})}{\partial x}=\mathbf{S}(\mathbf{U})
$$

by considering that the fields depend on time $t$ and one space coordinate only, namely $x$. Above, $\mathbf{U}$ represents the vector of the mixture fields, $\mathbf{F}(\mathbf{U})$ the flux vector and $\mathbf{S}(\mathbf{U})$ the vector of source contributions due to the chemical reactions, given by

$$
\mathbf{U}=\left(\begin{array}{l}
\rho_{1} \\
\rho_{2} \\
\rho v \\
\rho e
\end{array}\right) \equiv\left(\begin{array}{l}
u_{1} \\
u_{2} \\
u_{3} \\
u_{4}
\end{array}\right), \quad \mathbf{F}(\mathbf{U})=\left(\begin{array}{c}
\rho_{1} v \\
\rho_{2} v \\
\rho v^{2}+p \\
\rho e v+p v
\end{array}\right) \equiv\left(\begin{array}{c}
\frac{u_{1} u_{3}}{u_{1}+u_{2}} \\
\frac{u_{2} u_{3}}{u_{1}+u_{2}} \\
\frac{u_{3}^{2}}{u_{1}+u_{2}}+p \\
\frac{\left(u_{4}+p\right) u_{3}}{u_{1}+u_{2}}
\end{array}\right), \quad \mathbf{S}(\mathbf{U})=\left(\begin{array}{c}
\tau_{1} \\
\tau_{2} \\
0 \\
0
\end{array}\right)
$$

In particular, $\tau_{1}$ and $\tau_{2}$ represent the production rates of mass densities of the constituents associated to the chemical reaction. From the structural point of view, the above equations may follow from the Boltzmann equation or may be considered as the balance equations of a six-field theory of continuum thermodynamics for a reacting binary mixture. The pressure $p$, the internal energy density $\rho \varepsilon$ and the total energy density $\rho e$ of the mixture are given by

$$
p=\frac{k}{m} \rho T, \quad \rho \varepsilon=\frac{3}{2} p+n_{1} \mathrm{e}_{1}+n_{2} \mathrm{e}_{2}, \quad \rho e=\rho \varepsilon+\frac{\rho v^{2}}{2}
$$

where $T$ is the temperature of the mixture, $n_{\alpha}=\rho_{\alpha} / m$ the particle number density of the constituent $\alpha$, with $\alpha=1,2$, and $k$ the Boltzmann constant.

Chemical equilibrium for the reaction $A_{1}+A_{1} \rightleftharpoons A_{2}+A_{2}$ is characterized by the condition that the chemical potentials $\mu_{\alpha}$ of the constituents at equilibrium should obey the relationship

$$
\sum_{\alpha=1}^{2} v_{\alpha} \mu_{\alpha}^{\mathrm{eq}}=0, \quad v_{1}=-v_{2}=-1,
$$


where $v_{1}$ and $v_{2}$ are stoichiometric coefficients. The chemical potential for molecules which have internal variables associated with the formation energy - but not with the rotational, vibrational, electronic and nuclei states of the molecules - is given by

$$
\mu_{\alpha}=\mathrm{e}_{\alpha}+k T\left[\ln n_{\alpha}-\frac{3}{2} \ln (\mathcal{C} T)\right], \quad \alpha=1,2
$$

where it was supposed that the constituents have the same temperature of the mixture. Above $\mathcal{C}$ is a constant, which in statistical mechanics is identified with $\mathcal{C}=\left(2 \pi \mathrm{km} / h^{2}\right)^{3 / 2}$, where $h$ is Planck's constant. From the chemical equilibrium condition (4) we get the law of mass action in the form

$$
Q=2\left(\mathrm{e}_{2}-\mathrm{e}_{1}\right)=2 k T \ln \left(\frac{n_{1}^{\mathrm{eq}}}{n_{2}^{\mathrm{eq}}}\right),
$$

where $Q$ denotes the reaction heat. Note that the particle number densities in the above equation should be evaluated at equilibrium.

In order to close the system of equations (1) we have to know the source contributions due to the chemical reactions $\tau_{1}$ and $\tau_{2}$. For that end we rely on kinetic theory of reacting mixtures and write

$$
\tau_{1}=m\left[k_{r}(T) n_{2}^{2}-k_{f}(T) n_{1}^{2}\right]=-\tau_{2} .
$$

In the above equation $k_{r}(T)$ and $k_{t}(T)$ represent the reaction rate coefficients of the reverse and forward direction, respectively. These coefficients have been obtained from the Boltzmann equation by using a differential cross section with a barrier associated with the activation energy and resorting to the Maxwellian distribution functions which do not refer to the chemical equilibrium. They are given by

$$
k_{r}(T)=4 \sqrt{\frac{\pi k T}{m}} s^{2} \mathrm{~d}^{2} \exp \left(-\frac{\mathrm{e}_{r}}{k T}\right), \quad k_{f}(T)=4 \sqrt{\frac{\pi k T}{m}} s^{2} \mathrm{~d}^{2} \exp \left(-\frac{\mathrm{e}_{f}}{k T}\right),
$$

where $\mathrm{e}_{f}$ and $\mathrm{e}_{r}$ are the forward and reverse activation energies, respectively, and $s$ is the steric factor. The above expressions for the rate coefficients represent Arrhenius law. Note that the source contributions $\tau_{\alpha}$ due to the chemical reactions, given in Eq. (7), vanish at equilibrium, since the reaction heat is also given by the difference of the forward and reverse activation energies, i.e, $Q=\mathrm{e}_{f}-\mathrm{e}_{r}$, and the particle number densities at equilibrium are constrained to the mass action law (6). For the purpose of the next sections, we write the system of equations (1) in a more convenient form, namely

$$
\frac{\partial \mathbf{U}}{\partial t}+\mathbf{A}(\mathbf{U}) \frac{\partial \mathbf{U}}{\partial x}=\mathbf{S}(\mathbf{U}), \quad \text { where } \quad \mathbf{A}(\mathbf{U})=\frac{\partial \mathbf{F}(\mathbf{U})}{\partial \mathbf{U}},
$$

denotes the Jacobian matrix

$$
\mathbf{A}(\mathbf{U})=\left(\begin{array}{cccc}
\frac{\rho_{2}}{\rho} v & -\frac{\rho_{1}}{\rho} v & \frac{\rho_{1}}{\rho} & 0 \\
-\frac{\rho_{2}}{\rho} v & \frac{\rho_{1}}{\rho} v & \frac{\rho_{2}}{\rho} & 0 \\
-\frac{2}{3}\left(v^{2}+\frac{\mathrm{e}_{1}}{m}\right) & -\frac{2}{3}\left(v^{2}+\frac{\mathrm{e}_{2}}{m}\right) & \frac{4}{3} v & \frac{2}{3} \\
-v\left(\varepsilon+\frac{p}{\rho}+\frac{v^{2}}{6}+\frac{2}{3} \frac{\mathrm{e}_{1}}{m}\right) & -v\left(\varepsilon+\frac{p}{\rho}+\frac{v^{2}}{6}+\frac{2}{3} \frac{\mathrm{e}_{2}}{m}\right) & \varepsilon+\frac{p}{\rho}-\frac{v^{2}}{6} & \frac{5}{3} v
\end{array}\right) .
$$

\section{FIELD PERTURBATIONS FROM AN EQUILIBRIUM STATE}

We shall consider a small perturbation of the basic fields from an equilibrium state $\mathbf{U}_{0}$ characterized by constant mass densities $\rho_{1}^{0}, \rho_{2}^{0}$, constant total energy $\rho_{0} \varepsilon_{0}$ and vanishing velocity $v$, constrained to the mass action law (6), and then 
we expand the vector $\mathbf{U}$ of the mixture fields as

$$
\mathbf{U}=\mathbf{U}_{0}+\overline{\mathbf{U}}, \quad \text { with } \quad \mathbf{U}_{0}=\left(\begin{array}{c}
\rho_{1}^{0} \\
\rho_{2}^{0} \\
0 \\
\rho_{0} \varepsilon_{0}
\end{array}\right), \quad \overline{\mathbf{U}}=\left(\begin{array}{c}
\bar{u}_{1} \\
\bar{u}_{2} \\
\bar{u}_{3} \\
\bar{u}_{4}
\end{array}\right),
$$

where $\bar{u}_{1}$ through $\bar{u}_{4}$ are considered to be small perturbations to the equilibrium state fields.

The insertion of expansions (11) into the balance equations (9), together with the corresponding expansions of the matrices $\mathbf{A}(\mathbf{U})$ and $\mathbf{S}(\mathbf{U})$ about the equilibrium state, leads to

$$
\frac{\partial \overline{\mathbf{U}}}{\partial t}+\left[\mathbf{A}\left(\mathbf{U}_{0}\right)+\underline{\left.\left(\frac{\partial \mathbf{A}}{\partial \mathbf{U}}\right)_{0} \overline{\mathbf{U}}\right]} \frac{\partial \overline{\mathbf{U}}}{\partial x}=\mathbf{S}\left(\mathbf{U}_{0}\right)+\left(\frac{\partial \mathbf{S}}{\partial \mathbf{U}}\right)_{0} \overline{\mathbf{U}}\right.
$$

The underlined term in the above equation can be neglected, since we are taking into account only small perturbations about the equilibrium state, so that non-linear terms in the perturbations will be disregarded. As previously anticipated, the equilibrium value of the source contributions due to the chemical reactions vanishes, i.e., $\mathbf{S}\left(\mathbf{U}_{0}\right)=0$. Moreover, the matrices $\mathbf{A}\left(\mathbf{U}_{0}\right)$ and $(\partial \mathbf{S} / \partial \mathbf{U})_{0}$, omitting the index 0 in their elements for simplicity, read

$$
\begin{gathered}
\mathbf{A}\left(\mathbf{U}_{0}\right)=\left(\begin{array}{cccc}
0 & 0 & \frac{\rho_{1}}{\rho} & 0 \\
0 & 0 & \frac{\rho_{2}}{\rho} & 0 \\
-\frac{2}{3} \frac{e_{1}}{m} & -\frac{2}{3} \frac{e_{2}}{m} & 0 & \frac{2}{3} \\
0 & 0 & \varepsilon+\frac{p}{\rho} & 0
\end{array}\right), \\
\left(\frac{\partial \mathbf{S}}{\partial \mathbf{U}}\right)_{0}=\left(\begin{array}{cccc}
-2 \frac{\Delta}{\tau x_{1}}+\left(1+\frac{2}{3} \frac{\mathrm{e}_{1}}{k T}\right) \mathcal{Q} \frac{\Delta}{\tau} & 2 \frac{\Delta}{\tau x_{2}}+\left(1+\frac{2}{3} \frac{\mathrm{e}_{1}}{k T}+\frac{\mathcal{Q}}{3}\right) \mathcal{Q} \frac{\Delta}{\tau} & 0 & -\frac{2}{3} \frac{m \mathcal{Q}}{k T} \frac{\Delta}{\tau} \\
2 \frac{\Delta}{\tau x_{1}}-\left(1+\frac{2}{3} \frac{\mathrm{e}_{1}}{k T}\right) \mathcal{Q} \frac{\Delta}{\tau} & -2 \frac{\Delta}{\tau x_{2}}-\left(1+\frac{2}{3} \frac{\mathrm{e}_{1}}{k T}+\frac{\mathcal{Q}}{3}\right) \mathcal{Q} \frac{\Delta}{\tau} & 0 & \frac{2}{3} \frac{m \mathcal{Q}}{k T} \frac{\Delta}{\tau} \\
0 & 0 & 0 & 0 \\
0 & 0 & 0 & 0
\end{array}\right) .
\end{gathered}
$$

In Eq. (14) we have introduced the concentrations $x_{\alpha}=n_{\alpha} / n$, with $n=n_{1}+n_{2}$, the dimensionless reaction heat $\mathcal{Q}=Q / k T$, as well as the mean free time $\tau$ and the exponential factor $\Delta$ of the Arrhenius law defined by

$$
\tau=\frac{1}{4 n \mathrm{~d}^{2}} \sqrt{\frac{m}{2 \pi k T}}, \quad \Delta=x_{1}^{2} \frac{s^{2}}{\sqrt{2}} \exp \left(-\frac{\mathrm{e}_{f}}{k T}\right) .
$$

We note that, when the reaction heat vanishes and the activation energy $\mathbf{e}_{f} / k T$ has a very large value, so that the energy barrier is too high and the occurrence of a chemical reaction is practically nonexistent, the Jacobian matrix of the source term at equilibrium, $(\partial \mathbf{S} / \partial \mathbf{U})_{0}$, reduces to the null matrix.

The perturbation procedure adopted here assumes that the deviations from the equilibrium state can be studied at the level of an Eulerian regime describing the non-equilibrium effects due to the heat of the chemical reaction.

\section{Normal mode analysis}

Now let us suppose that the perturbations are related with plane harmonic waves of small amplitudes propagating in the $x$-direction through a chemically reacting binary mixture at rest, in an equilibrium state characterized by the vector 
$\mathbf{U}_{0}$ of the equilibrium mixture fields previously introduced, so that the field vector of the perturbations is given by

$$
\overline{\mathbf{U}}=\widetilde{\mathbf{U}} \exp [\imath(\kappa x-\omega t)],
$$

where $\tilde{\mathbf{U}}$ is the vector whose components represent small amplitudes of the waves induced in the mixture fields, while $\kappa$ and $\omega$ are the wavenumber and the angular frequency of the wave, respectively.

The insertion of expressions (16) into Eq. (12) leads to the following system of algebraic equations for the amplitudes $\widetilde{\mathbf{U}}$

$$
\left[\omega \mathbf{I}-\kappa \mathbf{A}\left(\mathbf{U}_{0}\right)-\imath \mathbf{H}\left(\mathbf{U}_{0}\right)\right] \widetilde{\mathbf{U}}=0,
$$

where I represents the four-order identity matrix and $\mathbf{H}\left(\mathbf{U}_{0}\right)=(\partial \mathbf{S} / \partial \mathbf{U})_{0}$ denotes the Jacobian matrix of the source term at equilibrium. i.e.,

The system of algebraic equations has a non-trivial solution if the determinant of the coefficient's matrix vanishes,

$$
\left|\omega \mathbf{I}-\kappa \mathbf{A}\left(\mathbf{U}_{0}\right)-{ }_{\imath} \mathbf{H}\left(\mathbf{U}_{0}\right)\right|=0 .
$$

This condition defines the dispersion relation of the harmonic waves which relates the wavenumber $\kappa$ and the angular frequency $\omega$.

\section{Sound propagation}

The physical problem of sound wave propagation can be studied starting from the previous normal mode description in terms of harmonic waves. In this case the angular frequency is real, $\omega \in \mathbb{R}$, and the wavenumber is complex, $\kappa \in \mathbb{C}$. The phase velocity of the wave $v_{p}$ and its absorption coefficient $\alpha$ are defined by

$$
v_{p}=\frac{\omega}{\Re(\kappa)}, \quad \alpha=\Im(\kappa),
$$

where $\Re(\kappa)$ and $\Im(\kappa)$ represent the real and imaginary parts of $\kappa$.

In the particular case on a non-reacting mixture, we obtain from Eq. (18) the corresponding dispersion relation as

$$
1-\frac{2}{3}\left(\frac{\kappa}{\omega}\right)^{2}\left[\varepsilon+\frac{p}{\rho}-\frac{\rho_{1} \mathrm{e}_{1}+\rho_{2} \mathrm{e}_{2}}{m \rho}\right]=0,
$$

whose solution leads to the following expressions for the phase velocity $v_{p}$ and the attenuation coefficient $\alpha$ :

$$
v_{p}= \pm c= \pm \sqrt{\frac{2}{3}\left[\varepsilon+\frac{p}{\rho}-\frac{\rho_{1} \mathrm{e}_{1}+\rho_{2} \mathrm{e}_{2}}{m \rho}\right]}= \pm \sqrt{\frac{5}{3} \frac{p}{\rho}}, \quad \alpha=0,
$$

Hence, in the absence of chemical reactions, the attenuation coefficient vanishes and there is no dispersion in the phase velocity, since it reduces to the sound speed $c$ of a single monatomic gas. This is a well known behavior of a non-reacting mixture of Eulerian fluids.

On the other hand, in the case of a reacting mixture, the dispersion relation that follows from Eq. (18) is given by

$$
(\omega \tau)\left(1-\Gamma^{2}\right)+\imath \frac{2 \Delta}{x_{1} x_{2}}\left[\left(1+\frac{x_{1} x_{2}}{6} \mathcal{Q}^{2}\right)-\left(1+\frac{x_{1} x_{2}}{10} \mathcal{Q}^{2}\right) \Gamma^{2}\right]=0,
$$

where $\omega \tau$ is a dimensionless oscillation frequency and $\Gamma=\kappa c / \omega$ is a dimensionless wavenumber. The solution of the above dispersion relation leads to

$$
\Gamma=\frac{c}{v_{p}}+l \frac{\alpha c}{\omega}= \pm \frac{\sqrt{\omega \tau+l \frac{2 \Delta}{x_{1} x_{2}}\left(1+\frac{x_{1} x_{2}}{6} \mathcal{Q}^{2}\right)}}{\sqrt{\omega \tau+l \frac{2 \Delta}{x_{1} x_{2}}\left(1+\frac{x_{1} x_{2}}{10} \mathcal{Q}^{2}\right)}} .
$$


In the low-frequency limit, the solution of the dispersion relation (22) is found by expanding the dimensionless wavenumber $\Gamma$ in power series of the dimensionless oscillation frequency $\omega \tau$, that is

$$
\Gamma=a_{0}+a_{1}(\omega \tau)+a_{2}(\omega \tau)^{2}+\ldots
$$

where $a_{0}, a_{1}, a_{2}, \ldots$ are complex expansion coefficients. By inserting expansion (24) into the dispersion relation (22) and equating equal powers of $\omega \tau$, we get

$$
\begin{aligned}
& a_{0}= \pm \frac{\left(1+\frac{x_{1} x_{2}}{6} \mathcal{Q}^{2}\right)^{1 / 2}}{\left(1+\frac{x_{1} x_{2}}{10} \mathcal{Q}^{2}\right)^{1 / 2}}, \quad a_{1}= \pm \imath \frac{\left(x_{1} x_{2}\right)^{2}}{60} \frac{\mathcal{Q}^{2}}{\Delta} \frac{1}{\left(1+\frac{x_{1} x_{2}}{6} \mathcal{Q}^{2}\right)^{1 / 2}\left(1+\frac{x_{1} x_{2}}{10} \mathcal{Q}^{2}\right)^{3 / 2}} \\
& a_{2}=\mp \frac{\left(x_{1} x_{2}\right)^{3}}{120} \frac{\mathcal{Q}^{2}}{\Delta^{2}} \frac{\left(1+\frac{3}{20} x_{1} x_{2} \mathcal{Q}^{2}\right)}{\left(1+\frac{x_{1} x_{2}}{6} \mathcal{Q}^{2}\right)^{3 / 2}\left(1+\frac{x_{1} x_{2}}{10} \mathcal{Q}^{2}\right)^{5 / 2}} .
\end{aligned}
$$

For small values of the dimensionless heat of reaction, the phase velocity and the attenuation coefficient in the lowfrequency limit can be written as

$$
\begin{aligned}
\frac{c}{v_{p}} & = \pm 1 \pm \frac{x_{1} x_{2}}{30} \mathcal{Q}^{2}\left(1-\frac{7}{60} x_{1} x_{2} \mathcal{Q}^{2}\right) \mp \frac{\left(x_{1} x_{2}\right)^{3}}{120} \frac{\mathcal{Q}^{2}}{\Delta^{2}}\left(1-\frac{7}{20} x_{1} x_{2} \mathcal{Q}^{2}\right)(\omega \tau)^{2}+\ldots, \\
\frac{\alpha c}{\omega} & = \pm \frac{\left(x_{1} x_{2}\right)^{2}}{60} \frac{\mathcal{Q}^{2}}{\Delta}\left(1-\frac{7}{30} x_{1} x_{2} \mathcal{Q}^{2}\right)(\omega \tau)+\ldots,
\end{aligned}
$$

respectively. Equations (26) and (27) give explicit expressions, in the low-frequency limit, for the phase velocity $v_{p}$ and attenuation coefficient $\alpha$ in terms of the dimensionless reaction heat $\mathcal{Q}$, constituent's concentrations $x_{1}, x_{2}$ and activation energy $\mathrm{e}_{f}$ through the exponential factor of Arrhenius law $\Delta$. As pointed out by Foch, Uhlenbeck and Fuentes Losa [12], in their paper concerning the propagation of sound waves in non-reactive binary gas mixtures, viscous and thermal-diffusion dissipative effects do not affect the phase velocity when the oscillation frequency goes to zero. However, we verify from (26) that dissipative effects due to chemical reactions affect the phase velocity even when $\omega \rightarrow 0$ showing that it is more important to take into account the reaction heat than viscosity, thermal conductivity and diffusion when we are dealing with a reactive binary mixture in the low-frequency regime.

\section{Eigenmodes}

The dynamical behaviour of small local wave disturbances induced by spontaneous internal fluctuations in the reacting binary mixture can also be studied from the previous normal mode analysis. The mixture eigenmodes are obtained by solving the dispersion relation (18) for the angular frequency $\omega$ as a function of the wavenumber $\kappa$. In this case the wavenumber is real, $\kappa \in \mathbb{R}$, and the the angular frequency is complex, $\omega \in \mathbb{C}$. The real part of the angular frequency $\omega$ describes the oscillation frequency of a small internal perturbation with wavenumber $\kappa$, while the imaginary part of $\omega$ describes the decay in time of the oscillation amplitude.

Let us introduce an an effective mean free path $\ell=\tau c$ and re-write the dispersion relation (18) in the form

$$
(\omega \tau)^{3}-(\kappa \ell)^{2}(\omega \tau)+l \frac{2 \Delta}{x_{1} x_{2}}\left[\left(1+\frac{x_{1} x_{2}}{6} \mathcal{Q}^{2}\right)(\omega \tau)^{2}-\left(1+\frac{x_{1} x_{2}}{10} \mathcal{Q}^{2}\right)(\kappa \ell)^{2}\right]=0
$$

Solving the dispersion relation (28) by expressing the dimensionless angular frequency $\omega \tau$ in terms of the dimensionless wavenumber $\kappa \ell$, we obtain three modes. These modes can be divided into two hydrodynamic modes, in which $\omega \tau$ tends to zero when $\kappa \ell$ goes to zero, and one kinetic mode, in which $\omega \tau$ tends to a non-vanishing constant value when $\kappa \ell$ goes to zero. This spectrum can be obtained in the small wavenumber limit by expanding the dimensionless angular frequency $\omega \tau$ in power series of the dimensionless wavenumber $\kappa \ell$ as

$$
\omega \tau=a_{0}+a_{1}(\kappa \ell)+a_{2}(\kappa \ell)^{2}+\ldots,
$$


where, again, $a_{0}, a_{1}, a_{2}, \ldots$ are complex expansion coefficients. By inserting expansion (29) into the dispersion relation (28) and equating equal powers of $\kappa \ell$, it follows two sets of values for the complex coefficients, namely

$$
\begin{aligned}
& a_{0}=0, \quad a_{1}= \pm \frac{\left(1+\frac{x_{1} x_{2}}{10} \mathcal{Q}^{2}\right)^{1 / 2}}{\left(1+\frac{x_{1} x_{2}}{6} \mathcal{Q}^{2}\right)^{1 / 2}}, \quad a_{2}=-\imath \frac{\left(x_{1} x_{2}\right)^{2}}{60} \frac{\mathcal{Q}^{2}}{\Delta} \frac{1}{\left(1+\frac{x_{1} x_{2}}{6} \mathcal{Q}^{2}\right)^{2}}, \\
& a_{0}=-l \frac{2 \Delta}{x_{1} x_{2}}\left(1+\frac{x_{1} x_{2}}{6} \mathcal{Q}^{2}\right), \quad a_{1}=0, \quad a_{2}=\imath \frac{\left(x_{1} x_{2}\right)^{2}}{30} \frac{\mathcal{Q}^{2}}{\Delta} \frac{1}{\left(1+\frac{x_{1} x_{2}}{6} \mathcal{Q}^{2}\right)^{2}} .
\end{aligned}
$$

Therefore we obtain

$$
\omega \tau= \pm \frac{\left(1+\frac{x_{1} x_{2}}{10} \mathcal{Q}^{2}\right)^{1 / 2}}{\left(1+\frac{x_{1} x_{2}}{6} \mathcal{Q}^{2}\right)^{1 / 2}}(\kappa \ell)-\imath \frac{\left(x_{1} x_{2}\right)^{2}}{60} \frac{\mathcal{Q}^{2}}{\Delta} \frac{1}{\left(1+\frac{x_{1} x_{2}}{6} \mathcal{Q}^{2}\right)^{2}}(\kappa \ell)^{2}+\ldots
$$

for the hydrodynamic modes, and

$$
\omega \tau=-l \frac{2 \Delta}{x_{1} x_{2}}\left(1+\frac{x_{1} x_{2}}{6} \mathcal{Q}^{2}\right)+l \frac{\left(x_{1} x_{2}\right)^{2}}{30} \frac{\mathcal{Q}^{2}}{\Delta} \frac{1}{\left(1+\frac{x_{1} x_{2}}{6} \mathcal{Q}^{2}\right)^{2}}(\kappa \ell)^{2}+\ldots
$$

for the kinetic mode. From Eq. (32) we verify that, in the small wavenumber limit, the two hydrodynamic modes are sound modes describing sound propagation in opposite directions parallel to the wavenumber with velocity

$$
\frac{\Re(\omega \tau)}{\kappa \tau}= \pm c \frac{\left(1+\frac{x_{1} x_{2}}{10} \mathcal{Q}^{2}\right)^{1 / 2}}{\left(1+\frac{x_{1} x_{2}}{6} \mathcal{Q}^{2}\right)^{1 / 2}} .
$$

From Eq. (33) we infer that the kinetic mode does not propagate, since its velocity is zero. However, its damping, defined by the imaginary part of the angular frequency, is non-zero for all values of the dimensionless wavenumber $\kappa \ell$. When $\kappa \ell$ goes to zero, the damping of the kinetic mode approaches the value

$$
\Im(\omega \tau)=-\frac{2 \Delta}{x_{1} x_{2}}\left(1+\frac{x_{1} x_{2}}{6} \mathcal{Q}^{2}\right) .
$$

Note that, in the absence of chemical reactions, the reaction heat $\mathcal{Q}$ vanishes and the coefficient $\Delta$ is equal to zero, so that, in the small wavenumber limit, the kinetic mode vanishes while the hydrodynamic modes propagate with velocities $\pm c$.

For small values of the dimensionless reaction heat $\mathcal{Q}$, the eigenmodes in the small wavenumber limit follow from Eqs. (32) and (33), yielding

$$
\begin{aligned}
& \omega \tau= \pm\left(1-\frac{x_{1} x_{2}}{30} \mathcal{Q}^{2}+\frac{\left(x_{1} x_{2}\right)^{2}}{200} \mathcal{Q}^{4}\right)(\kappa \ell)-\imath \frac{\left(x_{1} x_{2}\right)^{2}}{60} \frac{\mathcal{Q}^{2}}{\Delta}\left(1-\frac{x_{1} x_{2}}{3} \mathcal{Q}^{2}\right)(\kappa \ell)^{2}+\ldots, \\
& \omega \tau=-\imath \frac{2 \Delta}{x_{1} x_{2}}\left(1+\frac{x_{1} x_{2}}{6} \mathcal{Q}^{2}\right)+\imath \frac{\left(x_{1} x_{2}\right)^{2}}{30} \frac{\mathcal{Q}^{2}}{\Delta}\left(1-\frac{x_{1} x_{2}}{3} \mathcal{Q}^{2}\right)(\kappa \ell)^{2}+\ldots
\end{aligned}
$$

Equations (36) and (37) give explicit expressions, in the small wavenumer limit, for the hydrodynamic and kinetic eigenmodes in terms of the dimensionless reaction heat $\mathcal{Q}$, constituent's concentrations $x_{1}, x_{2}$ and activation energy $\mathrm{e}_{f}$ through the exponential factor of Arrhenius law $\Delta$. 


\section{CONCLUSIONS AND FUTURE PROSPECTS}

In this work we have developed a theoretical treatment of time dependent processes in a chemically reacting binary mixture described by the hydrodynamic reactive Euler equations derived from the Boltzmann equation. The kinetic origin of the reactive Euler equations is reflected in the production rates of mass densities of the constituents, which follow an Arrhenius rate law. The analysis here formulated refers to the propagation of plane harmonic waves of small amplitude propagating in the reactive mixture in both cases of forced waves (sound propagation) and free waves (eigenmodes). A normal mode approach is assumed for the perturbations of the mixture basic fields from an equilibrium state and a linear dynamics is derived for the investigation of the sound wave propagation and of the behavior of small local wave disturbances. The dispersion relation of the normal modes is derived showing an explicit dependence of the dispersion and absorption of forced and free waves on the heat of the chemical reaction mass concentrations of the constituents and activation energy through the exponential factor of Arrhenius law.

As a future prospect, the inclusion of viscosity, thermal conductivity and diffusion will be investigate as well as some numerical simulations will be implemented to test the theoretical treatment here developed, and some concrete examples will be explored. Comparisons with available experimental data and computational investigations will then be considered to test the theory and the numerics.

\section{ACKNOWLEDGMENTS}

The paper is partially supported by Brazilian Research Council (CNPq), by the Research Centre of Mathematics of the University of Minho through the FEDER Funds Programa Operacional Factores de Competitividade COMPETE and by the Portuguese Funds through the Fundação para a Ciência e Tecnologia, Project Est-C/MAT/UI0013/2011.

\section{REFERENCES}

1. V. Giovangigli, Multicomponent Flow Modeling, Birkhäuser, Boston, 1999.

2. E. Nagnibeda and E. Kustova, Non-Equilibrium Reacting Gas Flows: Kinetic Theory of Transport and Relaxation Processes, Springer Verlag, Berlin, 2009.

3. R. Brun, Introduction to reactive gas dynamics, Oxford University Press, New York, 2009.

4. G. M. Kremer, An introduction to the Boltzmann equation and transport processes in gases, Springer Verlag, Berlin, 2010.

5. L. S. Garcia-Colin and S. M. Y. de la Selva, "On the propagation of sound in chemically reacting fluids", Physica 75, 37-56 (1974).

6. J. P. Barton, "Sound propagation within a chemically reacting ideal gas", J. Acoust. Soc. Am. 81, 233-237 (1987).

7. L. Qin and J. S. Dahler, "The kinetic theory of a simple, chemically reactive fluid: scattering functions and relaxation processes", J. Chem. Phys. 103, 725-750 (1995).

8. W. Marques Jr., G. M. Alves and G. M. Kremer, "Light scattering and sound propagation in a chemically reacting binary gas mixture", Physica A 323, 401-412 (2003).

9. G. M. Alves, G. M. Kremer, W. Marques Jr. and A. J. Soares, "A kinetic model for chemical reactions without barriers: transport coefficients and eigenmodes", J. Stat. Mech. P03014, 1-20 (2011).

10. J. M. Shuen, M. S. Liou and B. van Leer, "Inviscid flux-splitting algorithms for real gases with non-equilibrium chemistry", $J$. Comput. Phys. 90, 371-395 (1990).

11. P. W. Atkins, Physical Chemistry, 5th. edition, Oxford University Press, Oxford, 1994.

12. J. D. Foch Jr., G. E. Uhlenbeck and M. F. Losa, "Theory of sound propagation in mixtures of monatomic gases", Phys. Fluids 15, 1224-1232 (1972). 